\title{
PSeUdo-Kümmel's Disease: A Unique Application for VERTEBROPLASTY
}

\author{
Joshua A. Hirsch, MD, Arra S. Reddy, MD, Italo Linfante, MD, and Jacob R. Rachlin, MD
}

\begin{abstract}
Compression fractures of the vertebrae are a major public health concern. There are approximately 700,000 compression fractures of the vertebrae diagnosed on an annual basis in the thoracolumbar spine. The etiology of senile osteoporosis is multifactorial with the most significant reason being agerelated bone loss. Multiple effects of compression fractures include acute and chronic pain syndromes, inability to perform activities of daily living, insomnia and depression.

Conventional treatment concepts relate to immobilization of the spine, medical pain control, bracing of the back and physical therapy. Vertebroplasty was developed in the
\end{abstract}

Osteoporosis is pervasive in the elderly. There are approximately 700,000 compression fractures of the vertebrae in the thoraco-lumbar spine annually $(1,2)$. This represents a major public health concern. The etiology of senile osteoporosis is multi-factorial with the most significant reason being age related bone loss. Other secondary associations are Caucasian or Asian race, thin body habitus, family history, early menopause, estrogen deficiency, sedentary lifestyle and smoking.

The effects of compression fracture are varied. They include acute and chronic pain syndromes, inability to perform activities of daily living, insomnia and depression. In addition, the loss of vertebral body height can result in kyphoscoliosis and may lead to functional disability and disfiguring syndromes.

From The Departments of Interventional Neuroradiology and Neurosurgery, Harvard University School of Medicine, Beth Israel Deaconess Medical Center in Boston, Massachusetts. Address Correspondence: Joshua Hirsch, MD, Interventional Neuroradiology, Beth Israel Deaconess Medical Center, One Deaconess Road, Boston, MA 02215. E-mail: jhirsch@bidmc.harvard.edu

No financial support was obtained in the completion of the study and preparation of the manuscript.
1980 as a treatment for painful cervical hemangiomas in France. Vertebroplasty has been utilized since 1993 to treat painful, osteoporotic compression fractures. In 1891, Kümmell described the disease with a posttraumatic osteitis in which patients developed a painful kyphosis after a period of being symptom free. Inferential evidence includes that vertebrae in this disease are being subjected to a form of avascular necrosis with intraosseous vacuum phenomenon. Patients with Kümmell's disease, treated with vertebroplasty, have been reported to do very well.

In a patient with an advanced case of vertebrae plana, without so intending, au-

thors placed air into the vertebral body and created so-called pseudo-Kümmell's disease. This case report describes with high risk or extreme vertebroplasty to alleviate symptomatology.

It is concluded that as the clinical experience with percutaneous vertebroplasty continues to expand, the approaches to treatment can become more focused on the specific disease state and specific treatment paradigms.

Keywords: Osteoporosis, thoracolumbar spine, Kümmell's disease, pseudo-Kümmell's disease, vertebroplasty

Conventional treatment concepts relate to immobilization of the spine and medical pain control. Immobilization led to prolonged bed-rest and bracing of the back. Medical pain control often required substantial narcotic loads and non-steroidal anti-inflammatory drugs (NSAIDs). Occasionally, physical therapy was tried but was often limited by pain and disability. Of course, each of the above treatments had its own associated morbidities. Prolonged immobilization leads to muscle atrophy with progressive debilitation and high narcotic requirements have even more obvious consequences.

The French developed percutaneous vertebroplasty in the 1980's as a treatment for painful cervical hemangiomas. In 1993, interventional neuroradiologists utilized vertebroplasty at the University of Virginia to treat a painful, osteoporotic compression fracture. The procedure was very successful in relieving the patient's pain. The University of Virginia became the pilot site for percutaneous vertebroplasty in this country. Over the next four years thirty patients were treated for a total of 48 compression fractures and this work was published in a landmark article in the 1997 American Journal of Neuroradiology (3).
Following presentation and publication of the original work from the University of Virginia group, multiple other series have pointed to the safety and efficacy of percutaneous vertebroplasty. These various studies report significant improvements in pain and mobility in as many as $95 \%$ of patients treated with this procedure $(4,5)$.

Most contraindications to the procedure are relative. These include intolerance of the prone position, coagulopathy, cord compression and vertebrae plana. The contraindications are for straightforward reasons. Vertebrae plana is contraindicated because of the technical difficulty in placing a needle into a vertebral body that has lost that much vertical height.

Typically, the authors utilize an active injection technique, i.e., following placement of the needle(s) polymethylmethacrylate (PMMA) is injected directly into the vertebral body. In fact, upon removal of the trocar, the needle is filled with saline prior to injection with PMMA. PMMA is then allowed to disperse through the osteoporosistic bone (6). In this case, as is described below, the authors initially inadvertently placed air and then actively injected a small amount of air. To our knowledge, there have been 
no previous reports of vertebroplasty using injection of air and we propose to term our experience with this case, pseudo-Kummel's disease.

\section{Case Report}

A 78-year-old white female with multiple medical problems including a history of hypothyroidism. She presented with seven months of excruciating back pain. The pain began rather suddenly and the patient describes the pain as wrapping around her chest and extending underneath her breast. The patient develops increased pain when she leaves the recumbent position and she can't stand for more than 30 minutes without having to lie down. She specifically relates that this is limited by back pain. Lying down improves the pain. The patient also relates a history of osteoporosis for which she has been treated aggressively including at various times Footman, Calcium, Vitamin D and calcium enriched multivitamins.

Physical examination demonstrated the patient to appear older than her ex-
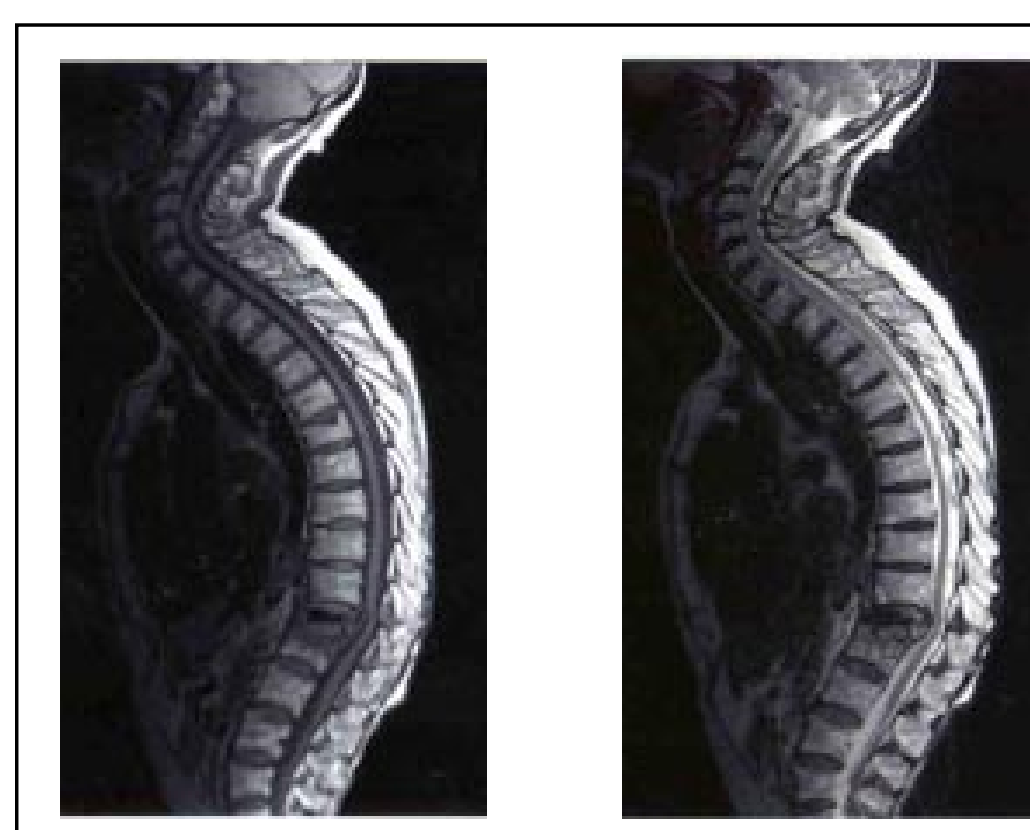

Fig. $1 \& 2$ These are MRI images obtained after the Vertebroplasty, however, they demonstrate some of the pre-vertebroplasty features. Both Fig 1 and 2 are sagittal MRI images of the spine obtained at 1.5 Tesla utilizing a phased array multicoil. This provides for excellent MRI imaging. Fig 1 is a short TR/short TE (T1 weighted) image of the cervicothoracic spine. Fig 2 is a long TR/long TE (T2 weighted) image of the same. Note the complete collapse of the vertebral body with effacement of the thecal sac and distortion of the lower thoracic cord. There is not definite signal abnormality within the cord. The extremely low intensity material seen in both images is the PMMA. normal appearing pedicles and the absence of metastatic disease in any vertebrae visualized, the lesion was felt to be an osteoporotic compression fracture rather than a malignant compression fracture.

Given the finding on physical/ neurologic examination and the MRI the patient was referred immediately to an experienced neurosurgeon (JRR) whose practice is largely composed of diseases of the spine. The patient was seen the same day by the neurosurgeon (JRR) who felt that the surgery to correct would possibly be too much for the patient to handle. He further felt that though the patient was showing signs of cord compression the major issue confronting her was pain. As such, he encouraged "high risk" vertebroplasty.

\section{Procedure}

The risks, benefits and alternatives to the procedure were explained to the patient, her husband and her daughters. They all understood the enhanced risks and gave written informed consent. The patient was brought to the neurointerventional operating theater and placed prone on the table. The procedure is done using a Phillips (Best, The Netherlands) BN5000 Allura biplane system ${ }^{\circledR}$. Procedure was performed using monitored anesthesia care. The aforementioned neurosurgeon (JRR) was available in the event of incipient decline.

As is our standard practice, 1 gram of intravenous Cefazolin was administered and a standard Betadine prep applied. The vertebral body was localized in the anteroposterior and lateral plane. One percent lidocaine was used for local anesthesia and delivered from the subcutaneous space to the level of the periosteum. A stab incision was made with a \#11 scalpel. A 13 gauge Jamshidi type needle was placed via a transpedicular approach though the left T 11 pedicle. However, it was placed at a slightly acute angle and bent while being placed. Because of the extreme nature of the plana it was decided to exchange the needle over an 035 Benson wire. While performing the exchange "air" was introduced into the vertebral body. The practitioners (JAH and ASR) elected to inject approximately $1-2 \mathrm{~mL}$ of additional air into the vertebral body. Following placement of the left sided needle a right-sided needle was placed following a similar outline to the process described above. After placing the needle it 

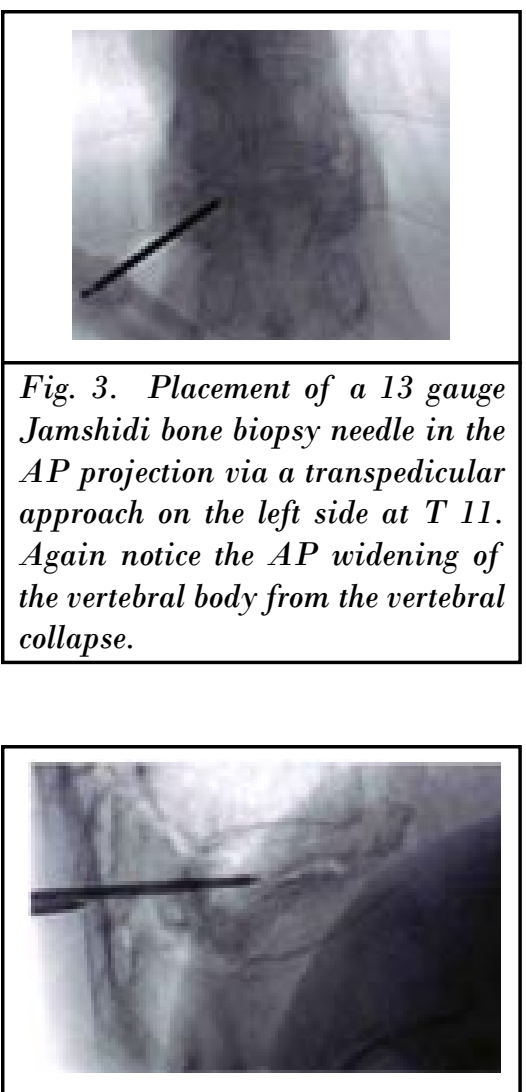

Fig6. Lateral projection demonstrating placement of the second Jamshidi needle. Again note the air which has been introduced into the vertebral body

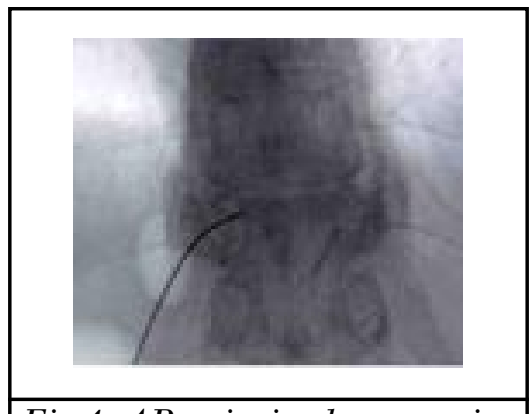

Fig. 4. AP projection demonstrating the 035 Benson wire over which the Jamshidi needle was exchanged.

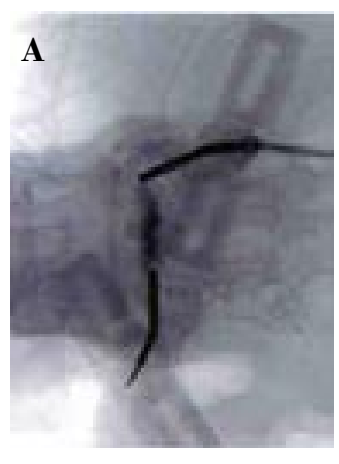

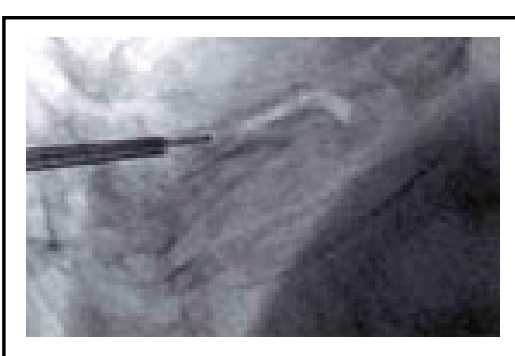

Fig.5 Lateral projection demonstrating the air (lucent area) within the vertebral body that was introduced initially during the over the wire exchange.

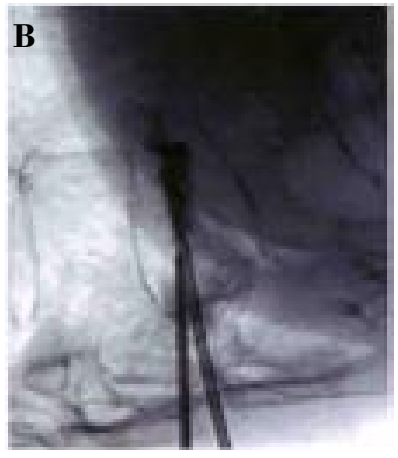

Fig 7 A-B. AP and lateral $X$-ray images prior to needle withdrawal demonstrating the PMMA filled vertebral body in the location of the previously seen air filled cavity. Note the PMMA does not extend into the posterior portion of the vertebral body on the lateral image.
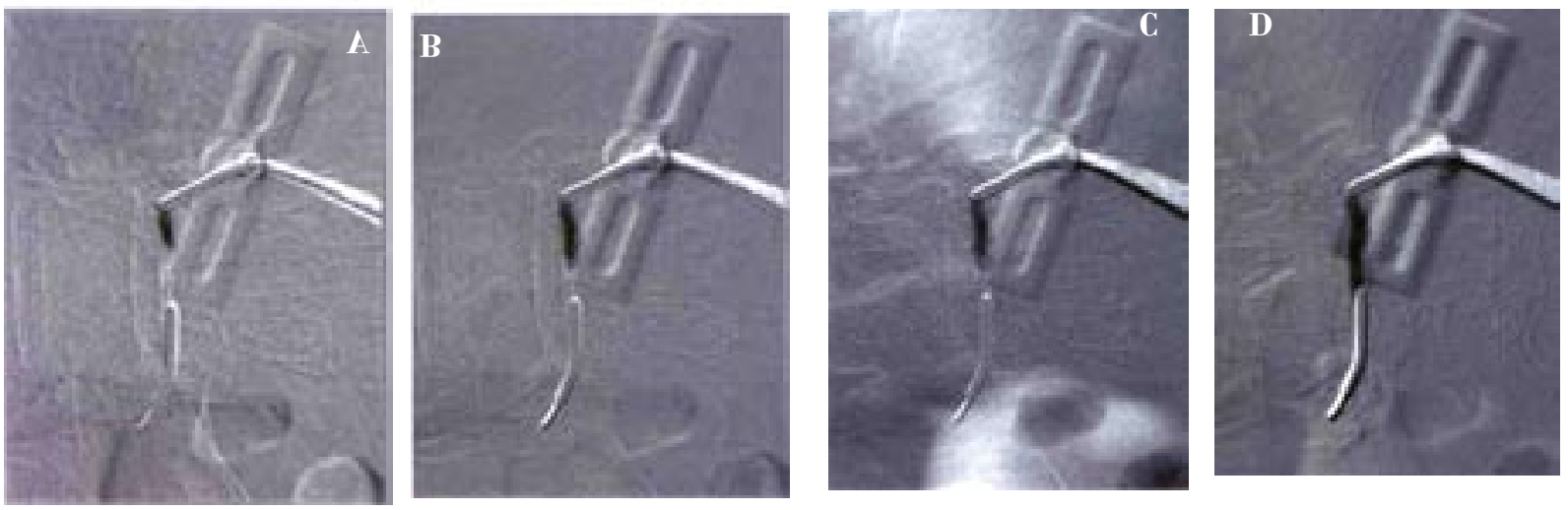

Fig.7A-D. A series of digitally subtracted AP images during PMMA injection. The air filled cystic cavity (demonstrated as the relatively lucent area) is gradually replaced with the dark material (PMMA mixed with radiotracer). Note the filling from the right sided injection of the entire vertebral body in the transverse projection. 


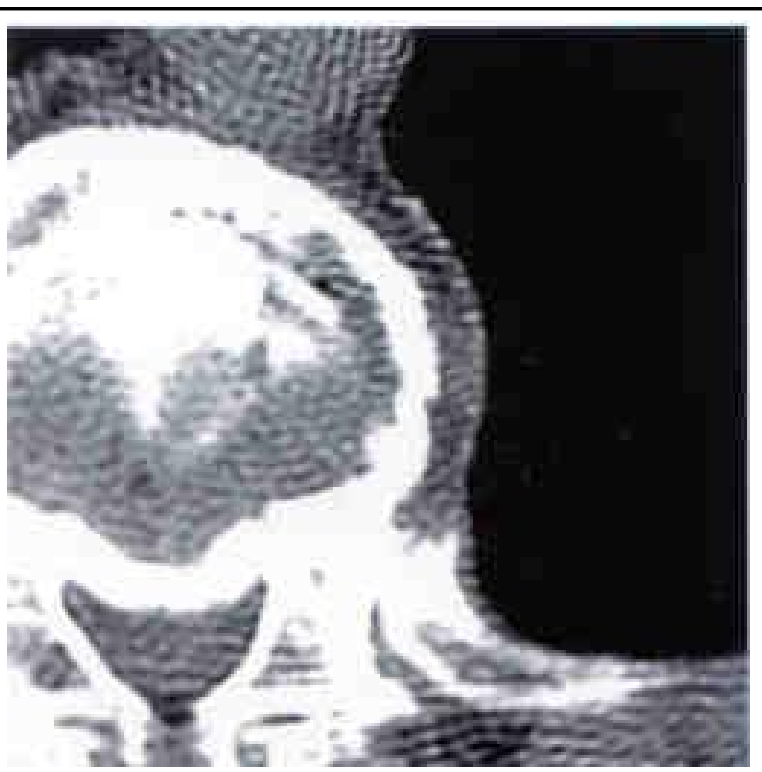

Fig 9. Immediate post- procedure CT scan demonstrating PMMA in the anterior portion of the vertebral body. Please note the posterior wall of the vertebral body and the distortion of the thecal sac.

appeared that the distal tip of the rightsided needle was slightly cephalad to the cystic cavity.

Polymethylmethacrylate was mixed in the standard fashion as suggested by the Parallax Company. This includes polymethylmethacrylate powder, polymethylmethacrylate solvent, injectable dexamethasone and radiotracer. The injector was hooked to the right sided needle (leur lock) and injection commenced.

Following completion of the PMMA injection both needles were removed and Syvek patches ${ }^{\circledR}$ (Marine Polymer Technologies; Danvers, MA) applied for hemostasis. The patient was transferred to the Post Anesthesia Care Unit. There were no complications.

\section{DISCUSSION}

Much of this material is contained in Resnick's comprehensive six volume orthopedic text. In 1891, Kümmell described the disease that now bears his name. He noted a post-traumatic osteitis in which patients developed a painful kyphosis after a period of being symptom free. He noted that this symptom free period could last months to years (7). Schmorl and Junghanns (8) developed pathologic correlates for the clinical findings. Though they were uncer- tain of the underlying disease their work supported the concept of delayed posttraumatic collapse of the vertebral bodies. This concept has been supported by numerous additional reports of the phenomena (9-13).

Ultimately, it is felt that the vertebrae is being subjected to a form of avascular necrosis. The evidence is in part inferential. This includes documentation of vascular occlusion by spinal arteriography (14) and the association with diseases that predispose to vascular difficulty.

The radiographic sine que non for Kümmell's disease is intraosseous vacuum phenomenon. That is to say, within the collapsed vertebrae air appears to be present. This is felt to be suggestive of ischemic necrosis $(15,16)$.

Magnetic Resonance diagnosis of Kümmell's is suggested by a cystic appearing lesion within the vertebral body. Often as a cystic cleft beneath a fracture. It is not certain how this relates to the previously described pathology associated with this disease. Recently, investigators described the MR imaging findings of these cystic clefts which depend on whether they are gas or liquid filled when they are imaged (17).

The relevance of these seeming esoteric distinctions to this patient were great. While it was not observed by Lane et al (18) in their work detailing their experience with intravertebral clefts, many practitioners believe that patients with socalled Kümmell's disease treated with vertebroplasty do very well. In this patient we created a Kümmell's like situation and hence have termed it Pseudo-Kümmell's. The patient had an advanced case of vertebra plana. Without so intending, we realized that we were placing "air" into the vertebral body as described above. By gingerly injecting further air we seemed to create a cystic cavity within the vertebral body.

The polymethylmethacrylate filled the cystic cavity, seeming to displace the air in the cystic cavity. This was well seen on both subtracted and unsubtracted images. Ultimately, polymethylmethacrylate regressed from the left sided needle. This was when the treatment was terminated.

In addition to being a radiographic curiosity, this case is important because most experienced vertebroplasty practitioners believe that the filling of the cystic cavity in Kummell's disease has a very high incidence of clinical improvement. In the author's experience the improvement in advanced cases of vertebra plana is typically less than complete, however, we believe that by creating this pseudocystic cavity within the bone the patient behaved in a fashion much more typical of Kümmell's disease.

While the technique had beneficial results in this case it needs to be viewed with caution. Specifically, the vertebral body is connected to the venous system. Early vertebroplasty practitioners, including the authors, would perform a "vertebrogram" by injecting the 11 gauge needle directly with iodinated contrast. This vertebrogram was, in essence, a vertebral body venogram. With such connections to the venous system the concern for air emboli to the lungs would always be present. Thus, we would not routinely advocate injecting air as part of a vertebroplasty. Circumstances permitted and then facilitated this unique application for this particular vertebral body in this patient.

\section{COnCLUsion}

The case represents an example of extreme vertebroplasty. As the clinical experience with percutaneous vertebroplasty continues to expand the approaches to treatment can become more focused on the specific disease state and specific treatment paradigms. 


\section{Author Affiliation:}

Joshua A. Hirsch, MD

Chief of Interventional Neuroradiology, Beth Israel Deaconess Medical Center, One Deaconess Road, Boston, MA 02215, phone (617) 7542521, fax (617) 754-2699, or e-mail: jhirsch@bidmc.harvard.edu

Arra S. Reddy, MD

Interventional Staff Radiologist, Department of Interventional Neuroradiology, Beth Israel Deaconess Medical Center, One Deaconess Road, Boston, MA 02215.

Italo Linfante, MD

Attending Stroke Neurologist, Beth Israel Deaconess Medical Center, One Deaconess Road, Boston, MA 02215

Jacob R. Rachlin, MD

Department of Neurosurgery, Beth Israel Deaconess Medical Center, One Deaconess Road, Boston, MA 02215

\section{REFERENCES}

1. Melton LJ III. How many women have osteoporosis now? J Bone Miner Res 1995; 10:175-177.

2. Melton LJ III, Kan SH, Frye MA et al. Epidemiology of vertebral fractures in women.
Am J Epidemiol 1989; 129:1000-1011.

3. Jensen ME, Evans AJ, Mathis JM et al. Percutaneous polymethylmethacrylate vertebroplasty in the treatment of osteoporotic vertebral body compression fractures: technical aspects. A/NR, 1997. 18: 1897-1904.

4. Grados F, Depriester C, Cayrolle G et al. Long-term observation of vertebral osteoporotic fractures treated by percutaneous vertebroplasty. Rheumatology 2000; 39: 1410-1414.

5. Martin JB, Jean B, Sugui K et al. Vertebroplasty; clinical experience and follow-up results. Bone 1999; 25:11S-15S.

6. Centenera LV, Choi IS, Hirsch JA. Percutaneous vertebroplasty treats compression fractures. Diagnostic Imaging 2000; 22: 147-153.

7. Stojanovic J, Kovac V. Diagnosis of ischemic vertebral collapse using skeletal spinal angiography. Fortschr Geb Rontgenstr Nuklearmed Erganzungsband 1981;10: 271-277.

8. Schmorl G, Junghanns $\mathrm{H}$. The Human Spine in Health and Disease. 2nd American Ed. Grune and Stratton (eds) New York, 1971; p 268.

9. Benedek TG, Nicholas JJ, Reece GJ. Kümmell's disease. A rare cause of post-traumatic back pain. Arthritis Rheum 1980; 23:653.

10. Brower AC, Downey EF Jr. Kümmell disease. Delayed collapse of the traumatized spine in a patient with Gaucher type 1 disease. Br J Radiol 1984; 57:833.
11. Hermann G, Goldblatt J, Desnick RJ. Kümmell disease: A delayed collapse of the traumatized spine in a patient with Gaucher type 1 disease. Br J Radiol 1984; 57: 833-835.

12. Nicholas JJ, Benedek TG, Reece GJ. Delayed traumatic vertebral body compression fracture; Part 1: clinical features. Semin Arthritis Rheum 1981; 10:2264-270.

13. Benedek TG, Nicholas JJ. Delayed traumatic vertebral body compression fracture: Part 2: Pathologic features. Semin Arthritis Rheum 1981; 10: 271-277.

14. Stojanovic J, Kovac V. Diagnosis of ischemic vertebral collapse using skeletal spinal angiography. Fortschr Geb Rontgenstr Nuklearmed Erganzungsband 1981; 10: 271-277.

15. Bretz GS, Jenkins PG. Gas density in an ununited fracture of a vertebral body. J Bone Joint Surg 1981; 63:1183-1184.

16. Maldague BE, Noel HM, Malghem JJ. The intravertebral vacuum cleft: A sign of ischemic vertebral collapse. Radiology 1978; 129:2329.

17. Naul LG, Peet GJ, Maupin WB. Avascular necrosis of the vertebral body: MR imaging. Radiology 1989; 172:219-222.

18. Lane JI, Maus TP, Wald JT et al. Intravertebral clefts opacified during vertebroplasty: pathogenesis, technical implications, and prognostic significance. Amer J Neurorad 2002; 23:1642-1646. 
